\title{
The Effect of Body Mass Index on in Vitro Fertilization/Intra-Cytoplasmic Sperm Injection Outcome in Egyptian Women with Polycystic Ovary Syndrome
} Original Article

\author{
Ahmad Mahran
}

Department of Obstetrics and Gynecology, Faculty of Medicine, Minia University, Egypt

\begin{abstract}
Objectives: To investigate the effect of body mass index (BMI) on outcome of IVF/ICSI in Egyptian women with PCOS.Study design: Retrospective cohort study.

Patients And Methods: The study included including 253 women with PCOS (study group) and 253 women with tubal or unexplained infertility (control group) that underwent IVF/ICSI treatment at Minia IVF Center in Egypt in the period between January 2013 and December 2015.

Results: The number of retrieved oocytes and the total number of embryos were significantly higher in women with PCOS compared to the control group ( $P=0.003$ and 0.002 respectively). There was no significant difference between the two groups as regards the clinical pregnancy rate (CPR), live birth rate and miscarriage rate. More cases of ovarian hyperstimulation syndrome (OHSS) were diagnosed in the PCOS group (24 vs. 5. $P=0.3$ ). When stratified for BMI, lean PCOS women $(\mathrm{BMI}<25)$ had higher CPR (35\% vs. $28.2, P=0.01)$, live birth rate $(32 \%$ vs. $26.1 \%, P=0.01)$. There were more cases of OHSS in lean as compared with overweight and obese PCOS women $(19 \%$ vs. $3.3 \%, P=0.05)$. Conclusion: Lean PCOS women achieved higher CPR and live birth rate in IVF/ICSI compared to overweight and obese PCOS women in Egyptian population.
\end{abstract}

Submitted: 9 November 2016, Accepted: 1 February 2017, Published: $x-x-x$

Key Words: Polycystic ovarian syndrome, body mass index, clinical pregnancy rate, live birth rate, ovarian hyperstimulation syndrome

Corresponding Author: Ahmad Mahran Department of Obstetrics and Gynecology, Faculty of Medicine, Minia University 61111 Minia, Egypt., E-mail: ezzeldin_ahmad@yahoo.com, Tel: 002-01115551073

ISSN: 2090-7265, February 2017, Vol. 7, No. 1

\section{INTRODUCTION}

Polycystic ovarian syndrome (PCOS) is the most common endocrinal disorder affecting women in the reproductive age. It accounts for $7080 \%$ - of cases of anovulatory infertility and a large proportion of these women undergo assisted reproductive technologies ${ }^{1,2}$.

Obesity is present in nearly half of women with $\mathrm{PCOS}^{3}$. It can initiate androgen production ${ }^{4}$ and obese girls are shown to exhibit higher levels of hyperandrogenaemia 5 . Obesity also exacerbates gonadotrophin and sex steroid secretion abnormalities and further down-regulate $\mathrm{SHBG}^{6}$. Women with PCOS demonstrate a high level of central adiposity even in those who are not obese ${ }^{7}$. The increased visceral fat is correlated to increased menstrual irregularities, Acanthosis nigricans and severe hirsutism ${ }^{8}$. The amount of visceral fat is shown to be related to the level of androgen ${ }^{9}$.
In non-PCOS women, BMI has been shown to affect IVF outcome ${ }^{10,11}$. However, in women with PCOs, the effect of BMI on IVF/ICSI outcome has not been fully evaluated. Few studies have tried to address this issue but their results were inconsistent ${ }^{12-16}$.

This study aimed to assess the effect of BMI on IVF/ ICSI outcome in Egyptian women with PCOS.

\section{PATIENTS AND METHODS}

This study was a retrospective cohort study including women with PCOS undergoing IVF/ICSI at Minia IVF center in the period between January 2013 and December 2015. A total of 253 women with PCOS were included. A non-random 1:1 age matched sample was selected from women with tubal or unexplained 
infertility that underwent IVF/ICSI treatment within the same period at the same center. Only the first cycle of each patient was included.

Diagnosis of PCOS was based on the Rotterdam criteria (17), in which at least two of the following three criteria were met: (a) oligo or anovulation, (b) clinical or biochemical hyperandrogenaemia, (c) polycytic ovaries $(>12$ follicles $<10 \mathrm{~mm}$ and/or ovarian volume $>10 \mathrm{ml}$ per ovary by vaginal ultrasound). Hyperprolactinaemia, thyroid dysfunction, Cushing's syndrome, congenital adrenal hyperplasia, an adrenal or ovarian tumor were excluded before enrollment in the study.

The exclusion criteria were: (a) Age $>39$ years, (b) FSH $>10$ IU/L. (c) Abnormal uterine cavity, (d) Surgically documented endometriosis, (e) Recurrent pregnancy loss, (f) Cases in whom testicular biopsies were used.

PCOS patients were stratified into two groups according to their BMI at the start of IVF/ICSI cycle:

- Group (A): Lean (BMI from 18.5 to $24.9 \mathrm{~kg} / \mathrm{m}^{2}$ ).

- Group (B): Overweight and obese (BMI $\left.\geq 25 \mathrm{~kg} / \mathrm{m}^{2}\right)$.

\section{Outcome measures}

- Clinical pregnancy: defined as evidence of fetal cardiac activity documented by ultrasound performed two weeks after a positive serum pregnancy test.

- Miscarriage: defined as loss of a clinical pregnancy prior to 24 weeks.

- Live birth: defined as the delivery of a viable infant.-

- Ovarian hyperstimulation syndrome (OHSS): defined as abdominal discomfort and bloating with ovarian enlargement that may be associated with decreased urine output. The condition is considered as severe OHSS if hospital admission is required for correction of haemoconcentration or electrolyte imbalance.

- Fertilization rate: defined as percentage of fertilized embryos to the number of mature oocytes.

- Implantation rate: defined as percentage of the number of gestational sacs documented by ultrasound to the number of transferred embryos.

This study was approved by the institutional review board of the faculty of Medicine, Minia University "as methodologically and ethically acceptable".

\section{Statistical analysis}

Statistical analysis was performed using Statistical Package for Social Science (SPSS Inc, Chicago) version 17 for Microsoft Windows. Data were described in terms of mean $\pm \mathrm{SD}$ (standard deviation) for continuous variables and frequencies (number of cases) and percentages for categorical data. Independent Student's t-test was used to compare quantitative variables and Chi square test was used to compare categorical data. $P<0.05$ was considered significant.

\section{RESULTS}

The current research included 253 women with PCOS and 253 age matched women with tubal or unexplained infertility. In the PCOS group153 patients were overweight and obese (BMI $\geq 25 \mathrm{~kg} / \mathrm{m}^{2}$ ) compared to 119 in the control group. Serum LH, AMH, antral follicle count (AFC) and ovarian volume were significantly higher in the PCOS group as compared with the control group. The total number of retrieved oocytes and embryos was higher in the PCOS group (18.1 \pm 4.7 vs. $11.7 \pm 4.5$, $P=0.03$ and11.12.8 \pm vs. $7.32 .6 \pm, P=0.02$ respectively). No significant difference obtained between two groups as regards the $\mathrm{CPR}$ and miscarriage rate and live birth rate. There was twenty four cases of OHSS in PCOS group compared to five cases in the control group $(P=0.3)$. The demographic features and the outcome measures in PCOS and control group are shown in Table 1.

Overweight and obese PCOs patients had more prevalent hyperandrogenism and less prevalent polycystic ovarian morphology on ultrasound as shown in Fig. 1.

In PCOS women, BMI was negatively correlated with AMH $(r=-0.52 ; P<0.001)$ and $\operatorname{AFC}(r=-0.48$, $P<0.001)$ as shown in Fig. 2 and 3. This correlation was not significant in the control group.

In lean PCOS patients, the duration of stimulation was shorter and the total dose of gonadotropins was lower as compared with overweight/obese patients. This difference was not observed in the control group. There was no significant difference as regards the number of oocytes retrieved, the number of mature oocytes, the total number of embryos and the number of grade-A embryos between lean and overweight/obese patients neither in PCOS nor in the control group. The details of stimulation cycles in the study population are shown in Table 2.

Implantation rate, CPR and live birth rates were higher is the lean PCOS patients as compared with overweight/ obese patients. On the other hand, there was inverse 
correlation between the incidence of OHSS and BMI. No significant difference obtained between the two groups as

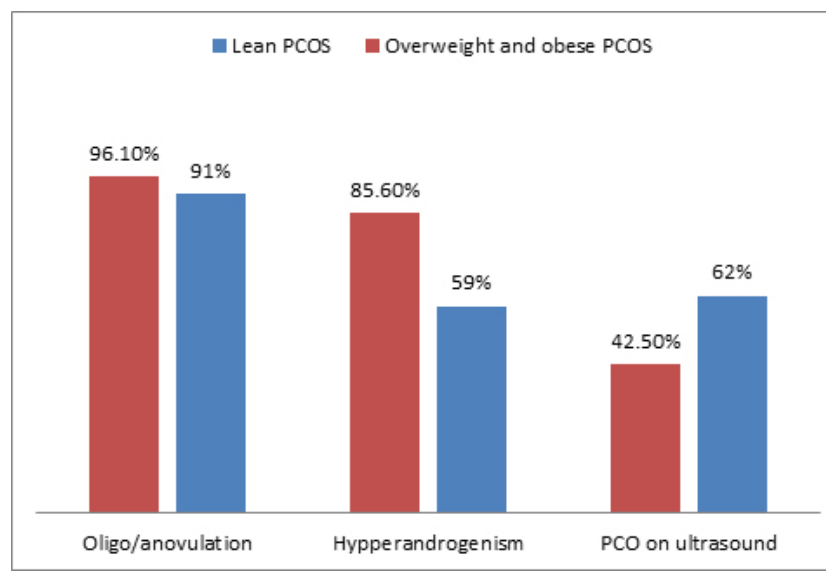

Fig. 1: Demographic characteristics of PCOS patients by BMI category.

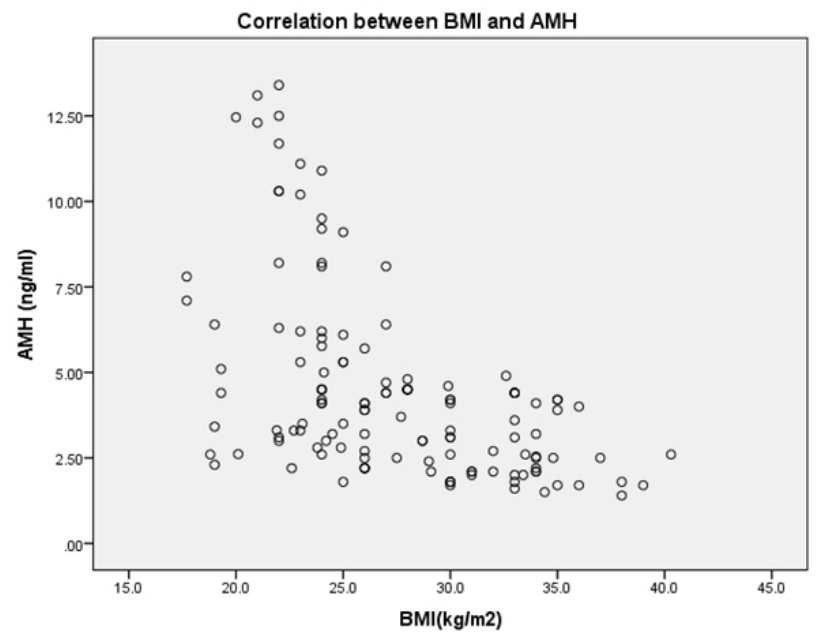

Fig. 2: Correlation between BMI and AMH

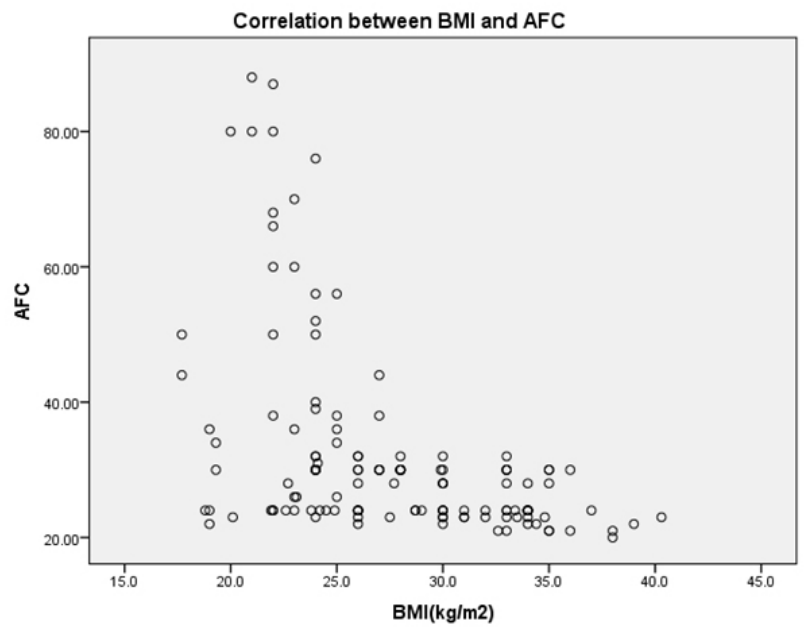

regards the fertilization and miscarriage rates. Outcome of the IVF/ICSI cycles in both groups shown in Table 3.

Table 1: Demographic characteristics and outcome in PCOS and the control group.

\begin{tabular}{lccc}
\hline & $\begin{array}{c}\text { PCOS } \\
(\mathbf{n}=\mathbf{2 5 3})\end{array}$ & $\begin{array}{c}\text { Tubal and } \\
\text { unexplained } \\
(\mathbf{n}=\mathbf{2 5 3})\end{array}$ & P value \\
\hline Age(years) & $27.3 \pm 2.9$ & $\mathrm{NS}$ & $29.2 \pm 3.1$ \\
\hline BMI (kg/m²) & $29.8 \pm 3.5$ & $0.01^{*}$ & $24.6 \pm 2.8$ \\
\hline Duration of infertility & $3.5 \pm 0.7$ & $\mathrm{NS}$ & $3.7 \pm 0.6$ \\
\hline FSH & $5.7 \pm 1.2$ & $\mathrm{NS}$ & $5.8 \pm 1.2$ \\
\hline LH & $10.5 \pm 1.6$ & $0.001^{*}$ & $4.9 \pm 1.1$ \\
\hline AMH & $7.2 \pm 1.7$ & $0.001^{*}$ & $3.5 \pm 0.9$ \\
\hline AFC & $35.3 \pm 4.4$ & $0.001^{*}$ & $14.2 \pm 2.3$ \\
\hline Ovarian volume & $11.2 \pm 1.6$ & $0.002^{*}$ & $6.2 \pm 0.8$ \\
\hline Total no. of & $18.1 \pm 4.7$ & $0.003^{*}$ & $11.7 \pm 4.5$ \\
\hline retrieved oocytes & $11.1 \pm 2.8$ & $0.002^{*}$ & $7.3 \pm 2.6$ \\
\hline Total no. of embryos & $81(32 \%)$ & $\mathrm{NS}$ & $92(36.4 \%)$ \\
\hline Clinical pregnancy & $8(3.2 \%)$ & $\mathrm{NS}$ & $5(2 \%)$ \\
\hline Miscarriage & $72(28.5 \%)$ & $\mathrm{NS}$ & $87(34.4 \%)$ \\
\hline Live birth & $24(9.5 \%)$ & $0.03^{*}$ & $5(2 \%)$ \\
\hline OHSS & & & \\
\hline
\end{tabular}

BMI: Body mass index

AMH: Anti-müllerian hormone

AFC: Antral follicle count

OHSS: Ovarian hyperstimulation syndrome

*Statistically significant.

Data is presented as mean $\pm \mathrm{SD}$ or frequency and percentages.

Fig. 3: Correlation between BMI and AFC. 
Table 2: Details of controlled stimulation cycles in the study population.

\begin{tabular}{|c|c|c|c|c|c|c|}
\hline & \multicolumn{2}{|c|}{ PCOS } & \multirow{2}{*}{$P$ value } & \multicolumn{2}{|c|}{ Tubal and unexplained } & \multirow[b]{2}{*}{$P$ value } \\
\hline & $\begin{array}{l}\text { BMI <25 } \\
(n=100)\end{array}$ & $\begin{array}{c}B M I \geq 25 \\
(n=153)\end{array}$ & & $\begin{array}{c}\mathrm{BMI}<25 \\
(\mathrm{n}=134)\end{array}$ & $\begin{array}{c}B M I \geq 25 \\
(n=119)\end{array}$ & \\
\hline \multicolumn{7}{|l|}{ Down-regulation protocol: } \\
\hline - long agonist & $53(53 \%)$ & $80(52.3 \%)$ & NS & $121(90.3 \%)$ & $101(84.9 \%)$ & NS \\
\hline - Antagonist & $47(47 \%)$ & $73(47.7 \%)$ & NS & $13(9.7 \%)$ & $18(15.1 \%)$ & NS \\
\hline Duration of stimulation & $11.5 \pm 3.2$ & $13.2 \pm 3.5$ & $0.03 *$ & $10.3 \pm 2.1$ & $10.7 \pm 2.2$ & NS \\
\hline Total dose of gonadotropins & $2215 \pm 455$ & $3105 \pm 545$ & $0.01 *$ & $2135 \pm 465$ & $2535 \pm 615$ & NS \\
\hline Total no. of retrieved oocytes & $18.3 \pm 5.2$ & $17.8 \pm 4.9$ & NS & $12.2 \pm 3.1$ & $11.5 \pm 2.8$ & NS \\
\hline No. of mature oocytes & $13.4 \pm 3.1$ & $13.1 \pm 3.1$ & NS & $9.5 \pm 2.8$ & $9.2 \pm 2.7$ & NS \\
\hline Total no. of embryos & $11.1 \pm 2.5$ & $10.9 \pm 2.6$ & NS & $7.5 \pm \pm 1.8$ & $7.1 \pm 1.9$ & NS \\
\hline No. of grade A embryos & $9.8 \pm 2.1$ & $9.7 \pm 1.8$ & NS & $7.1 \pm 1.5$ & $6.8 \pm$ & NS \\
\hline
\end{tabular}

Data is presented as mean $\pm \mathrm{SD}$ or frequency and percentages.

Table 3: Cycle outcome in PCOS and control group stratified by BMI.

\begin{tabular}{|c|c|c|c|c|c|c|}
\hline & \multicolumn{2}{|c|}{ PCOS } & \multirow{2}{*}{$P$ value } & \multicolumn{2}{|c|}{ Tubal and unexplained } & \multirow{2}{*}{$P$ valuc } \\
\hline & $\begin{array}{l}\mathrm{BMI}<25 \\
(\mathrm{n}=100)\end{array}$ & $\begin{array}{c}\mathrm{BMI} \geq 25 \\
(n=153)\end{array}$ & & $\begin{array}{c}\text { BMI<25 } \\
(n=134)\end{array}$ & $\begin{array}{c}B M I \geq 25 \\
(n=119)\end{array}$ & \\
\hline Fertilization rate & $82.8 \%$ & $83.2 \%$ & NS & $84.5 \%$ & $83.7 \%$ & NS \\
\hline Implantation rate & $37.2 \%$ & $31.4 \%$ & & $42.5 \%$ & $39.7 \%$ & NS \\
\hline Clinical pregnancy & $35(35 \%)$ & $46(28.2 \%)$ & $0.03^{*}$ & $52(38.8 \%)$ & $40(33.6 \%)$ & NS \\
\hline Miacarriage & $3(3 \%)$ & $5(3.3 \%)$ & $0.01^{*}$ & $2(1.5 \%)$ & $3(1.6 \%)$ & NS \\
\hline Live birth & $32(32 \%)$ & $40(26.1 \%)$ & NS & $50(37.3 \%)$ & $37(31.1 \%)$ & NS \\
\hline OHSS & $19(19 \%)$ & $5(3.3 \%)$ & $0.01^{*}$ & $3(2.2 \%)$ & $2(1.7 \%)$ & NS \\
\hline - Mild & $14(14 \%)$ & $3(3.3 \%)$ & $0.04^{*}$ & $3(2.4 \%)$ & $2(1.7 \%)$ & \\
\hline - Moderate & $3(3 \%)$ & $2(1.3 \%)$ & & 0 & 0 & \\
\hline - Severe & $2(2 \%)$ & 0 & & 0 & 0 & \\
\hline
\end{tabular}

Data is presented as number and percentages

\section{DISCUSSION}

Many studies have investigated the impact of BMI on the outcome of assisted reproductive technologies. Few of them have focused on patients with PCOS in whom overweight and obesity are commonly associated. Moreover, the results of these studies were controversial and inconsistent. PCOS is a heterogeneous condition and genetic factors may play a role in the pathogenesis. Furthermore, the distribution of the clinical, biochemical and ultrasound characteristics can differ among different races. To our knowledge, this is the first research to investigate the effect of BMI on the clinical and embryological outcome of IVF/ICSI among Egyptian women with PCOS. In the current study, we compared the IVF/ICSI outcome in 253 women with PCOS to 253 women with tubal or unexplained infertility "as control group". The patients in each group were stratified into two categories based on their BMI. As expected, patients with PCOS had higher BMI, serum LH levels, serum AMH levels, AFC and mean ovarian volume as compared with the control group. 
When stratified by BMI, lean PCOS women required lower dose of gonadotropins than the overweight and obese. There was no significant difference between lean and obese PCOS women as regards the number of retrieved oocytes, the number of mature oocytes or number of good quality embryos. However, lean women achieved higher CPR and live birth rate compared to the overweight and obese women. It is assumed that obesity alters the follicular androgen profile, insulin resistance and is associated with elevated leptin levels which inversely affect oocyte size, oocyte and embryo quality; changes that may be responsible for the poor outcome in overweight and obese PCOS women ${ }^{18,19}$.

There are limited studies linking BMI and PCOS. Moreover, their results are controversial, as Beydoun and colleagues reported no significant effect of BMI and outcome measures of infertility treatment success ${ }^{20}$. On the contrary, Orievto et al obtained a significantly higher CPR in PCOS patients with BMI $\leq 25 \mathrm{~kg} / \mathrm{m}^{2}$ compared to those with $\mathrm{BMI}>25 \mathrm{~kg} / \mathrm{m}^{215,21}$.

A recent Chinese study recruited 409 normal weight women with PCOS and 270 overweight and obese women revealed a higher implantation rate in lean PCOS women with no significant difference in CPR compared to overweight and obese women ${ }^{21,22}$. Higher miscarriage rate in obese PCOS women was reported by Ozgun et $\mathrm{al}^{16}$. On the other hand, similar miscarriage rates were shown by Huang et $\mathrm{al}^{22}$.

We found a higher incidence of OHSS in lean PCOS women as compared with overweight and obese women consistent with the reported by Bailey et $\mathrm{al}^{23}$, who hypothesized that BMI has an impact on the development of OHSS. They suggested that obese PCOs might have diminished ovarian reserve. In favor of this suggestion, an inverse correlation between BMI and $\mathrm{AMH}$ was demonstrated in the current study which supports the assumption that obesity can be linked to poor ovarian reserve.

The strengths of our study are the large number included and reporting of parameters like AMH and AFC which can be useful in better understanding of the relation of BMI to other clinical, biochemical and sonographic characteristics of PCOS women. The limitations include the retrospective nature of the study and reporting data from fresh cycle only so the cumulative live birth rate was not assessed.

\section{CONCLUSION}

Overweight and obese PCOS women achieved unfavorable outcome in IVF/ICSI compared to lean PCOS women. Subsequently, should be encouraged to lose weight prior to IVF/ICSI to improve the success rate.

\section{CONFLICT OF INTEREST}

No external fund was received for this study. .

\section{REFERENCES}

1. Asuncion M, Calvo RM, San Milan JL, Sancho J, Avila S, Escobar-Morreale HF. A prospective study of the prevalence of polycystic ovary syndrome in unselected Caucasian women from Spain. J Clin Endocrinol Metab 2000; 85(7): 2434 -2438.

2. Azziz R, Woods KS, Reyna R, Key TJ, Knochenhauer ES, YildizBO. The prevalence and features of the polycystic ovary syndrome in an unselected population. J Clin Endocrinol Metab 2004; 89(6): $2745-9$.

3. Balen A H, Conway G S, homburg R and Legro R S. Polycystic ovary syndrome: A guide to clinical management. London: Taylor\& Francis, 2005.

4. Broekmans FJ, Knauff EA, Valkenburg O, Laven JS, Eijkemans MJ, Fauser BC. PCOS according to the Rotterdam consensus criteria: Change in prevalence among WHO-II anovulation and association with metabolic factors. BJOG 2006; 113(10): 1210- 1217.

5. McCartney CR, Prendergast KA, Chhabra S, Eagleson CA, Yoo R, Chang RJ, et al. The association of obesity and hyperandrogenemia during the pubertal transition in girls: Obesity as a potential, factor in the genesis of postpubertalhyperandrogenism. J Clin Endocrinol Metab 2006; 91(5): 1714- 1722.

6. Lukanova A, Lundin E, Micheli A, Arslan A, Ferrari P, Rinaldi S, et al. Circulating levels of sex steroid hormones and risk of endometrial cancer inpostmenopausal women. Int J Cancer 2004; 108(3): $425-432$.

7. Kirchengast S, Huber J. Body composition characteristics and body fat distribution in lean women with polycystic ovary syndrome. Hum Reprod 2001; 16(6): 1255 -1260.

8. Pasquali R, Casimirri F, Venturoli S, Antonio M, Morselli L, Reho S, et al. Body fat distribution has weight-independent effects on clinical, hormonal, and metabolic features of women with polycystic ovary syndrome. Metabolism 1994; 43(6): 706 -713.

9. Stanley T, Misra M. Polycystic ovary syndrome in obese adolescents. Curr Opin Endocrinol Diabetes Obes 2008; 15(1): 306-. 
10. Moragianni VA, Jones SM, Ryley DA. The effect of body mass index on the outcomes of first assisted reproductive technology cycles. Fertil Steril 2012; 98(1): $102-108$.

11. Shah DK, Missmer SA, Berry KF, Racowsky C, Ginsburg ES. Effect of obesity on oocyte and embryo quality in women undergoing in vitro fertilization. Obstet Gynecol 2011; 118(1): 63- 70.

12. Beydoun HA, Stadtmauer L, Beydoun MA, Rus $\neg$ sell H, Zhao Y, Oehninger S. Polycystic ova $\neg$ ry syndrome, body mass index and outcomes of assisted reproductive technologies. Reprod Biomed Online 2009; 18(6): 856 -863.

13. McCormick B, Thomas M, Maxwell R, Williams D, Aubuchon M. Effects of polycystic ovarian syndrome on in vitro fertilization-embryo transfer outcomes are influenced by body mass index. Fertil Steril 2008; 90(6): $2304-2309$.

14. Jungheim ES, Lanzendorf SE, Odem RR, Moley $\mathrm{KH}$, Chang AS, Ratts VS. Morbid obesity is associated with lower clinical pregnancy rates after in vitro fertilization in women with poly $\neg$ cystic ovary syndrome. Fertil Steril 2009; 92(1): 256 -261

15. Orvieto R, Nahum R, Meltcer S, Homburg R, Rabinson J, Anteby EY, et al. Ovari $\neg$ an stimulation in polycystic ovary syndrome patients: The role of body mass index. Reprod Biomed Online 2009; 18(3): 333- 336.

16. Ozgun MT, Uludag S, Oner G, Batukan C, Aygen EM, Sahin Y. The influence of obesity on ICSI outcomes in women with polycystic ovary syndrome. J Obstet Gynaecol 2011; 31(3): 245 -249.

17. The Rotterdam ESHRE/ASRM-sponsored PCOS consensusworkshop group, Fauser B, Tarlatzis B, Chang J, Azziz R, et al. Consensus on diagnostic criteria and long-term health risks related to polycystic ovary syndrome (PCOS). Hum Reprod 2003;2004; 19(1): 41-7.
18. Marquard KL, Stephens SM, Jungheim ES, Ratts VS, Odem RR, Lanzendorf S, et al. Polycystic ovary syndrome and maternal obesity affect oocyte size in in vitro fertiliza $\neg$ tion/intracytoplasmic sperm injection cycles. Fertil Steril 2011; 95: 2145-2149.e1.

19. Shah DK, Missmer SA, Berry KF, Racowsky C, Ginsburg ES. Effect of obesity on oocyte and embryo quality in women undergoing in vitro fertilization. Obstet Gynecol 2011; 118(1): 63 -70.

20. Beydoun HA, Stadtmauer L, Beydoun MA, Rus $\neg$ sell H, Zhao Y, Oehninger S. Polycystic ova $\neg$ ry syndrome, body mass index and outcomes of assisted reproductive technologies. Reprod Biomed Online 2009; 18(6): $856-863$.

21. Zhiqin $\mathrm{Bu}$, Wei Dai, YihongGuo, Yingchun $\mathrm{Su}$, Jun Zhai, Yingpu Sun. Overweight and obesity adversely affect outcomes of assisted reproductive technologies in polycystic ovary syndrome patients Int J Clin Exp Med 2013; 6(10): 991- 995.

22. Huang $\mathrm{K}$, Liao $\mathrm{X}$, Dong $\mathrm{X}$, Zhang $\mathrm{H}$. Effect of overweight/obesity on IVF-ET outcomes in chinese patients with polycystic ovary syndrome. Int J Clin Exp Med 2014; 7(12): 5872 -5876.

23. Bailey AP, Hawkins LK, Missmer SA, Correia KF, Yanushpolsky EH. Effect of body mass index on in vitro fertilization outcomes in women with polycystic ovary syndrome. Am J Obstet Gynecol 2014; 211(2): 163.e1163-.e6. 